Article

\title{
Certain Unified Integrals Involving a Multivariate Mittag-Leffler Function
}

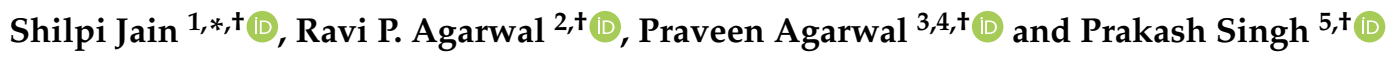 \\ 1 Department of Mathematics, Poornima College of Engineering, Jaipur 302022, India \\ 2 Department of Mathematics, Texas A\&M University, Kingsville, TX 78363, USA; Ravi.Agarwal@tamuk.edu \\ 3 Department of Mathematics, Anand International College of Engineering, Jaipur 303012, India; \\ goyal.praveen2011@gmail.com or praveen.agarwal@anandice.ac.in \\ 4 Nonlinear Dynamics Research Center (NDRC), Ajman University, Ajman 346, United Arab Emirates \\ 5 Department of Mathematics, Research Scholar, Poornima University, Jaipur 302022, India; \\ prakash.singh@anandice.ac.in or phdodd2018prakash6673@poornima.edu.in \\ * Correspondence: shilpijain1310@gmail.com or shilpi.jain@poornima.org \\ + These authors contributed equally to this work.
}

check for

updates

Citation: Jain, S.; Agarwal, R.P.; Agarwal, P.; Singh, P. Certain Unified Integrals Involving a Multivariate Mittag-Leffler Function. Axioms 2021, 10, 81. https://doi.org/10.3390/ axioms10020081

Academic Editor: Hans J. Haubold

Received: 23 March 2021

Accepted: 23 April 2021

Published: 2 May 2021

Publisher's Note: MDPI stays neutral with regard to jurisdictional claims in published maps and institutional affiliations.

Copyright: (c) 2021 by the authors. Licensee MDPI, Basel, Switzerland. This article is an open access article distributed under the terms and conditions of the Creative Commons Attribution (CC BY) license (https:/ / creativecommons.org/licenses/by/ $4.0 /)$.
Abstract: A remarkably large number of unified integrals involving the Mittag-Leffler function have been presented. Here, with the same technique as Choi and Agarwal, we propose the establishment of two generalized integral formulas involving a multivariate generalized Mittag-Leffler function, which are expressed in terms of the generalized Lauricella series due to Srivastava and Daoust. We also present some interesting special cases.

Keywords: generalized hypergeometric function ${ }_{p} F_{q}$; generalized (Wright) hypergeometric functions ${ }_{p} \Psi_{q}$; generalized Lauricella series; Oberhettinger's integral formula; generalized Mittag-Leffler function

MSC: primary 33B10; 33B15; 33C05; 33E12 secondary 33C20; 33C65

\section{Introduction and Preliminaries}

The generalized Lauricella series (see, for example, Refs. [1] (p. 454) and [2] (p. 37)) is defined as follows:

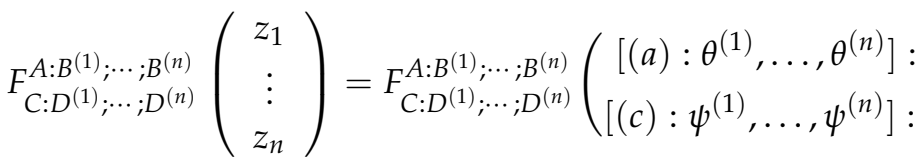

$$
\begin{aligned}
& \begin{array}{l}
{\left[(b)^{(1)}: \phi^{(1)}\right] ; \ldots ;\left[(b)^{(n)}: \phi^{(n)}\right] ;} \\
{\left[(d)^{(1)}: \delta^{(1)}\right] ; \ldots ;\left[(d)^{(n)}: \delta^{(n)}\right] ;}
\end{array} \\
& =\sum_{k_{1}, \ldots, k_{n}=0}^{\infty} \Omega\left(k_{1}, \ldots, k_{n}\right) \frac{z_{1}^{k_{1}}}{k_{1} !} \cdots \frac{z_{n}^{k_{n}}}{k_{n} !}
\end{aligned}
$$

where, for convenience,

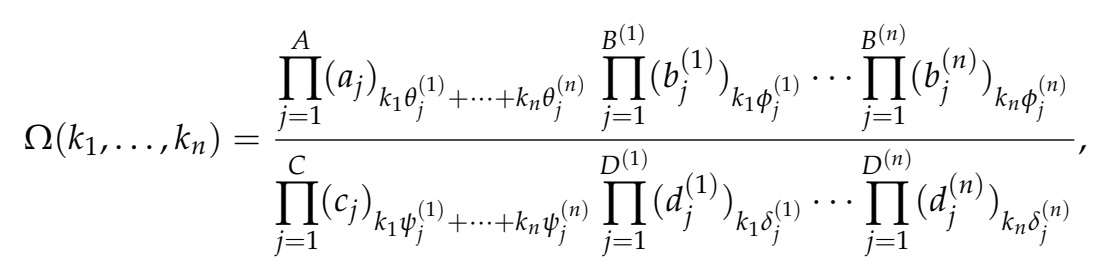

the coefficients 


$$
\left\{\begin{array}{l}
\theta_{j}^{(m)}(j=1, \ldots, A) ; \phi_{j}^{(m)}\left(j=1, \ldots, B^{(m)}\right) ; \\
\psi_{j}^{(m)}(j=1, \ldots, C) ; \delta_{j}^{(m)}\left(j=1, \ldots, D^{(m)}\right) ; \forall m \in\{1, \ldots, n\}
\end{array},\right.
$$

are real and positive, $(a)$ abbreviates the array of $A$ parameters $a_{1}, \ldots, a_{A}$, and $\left(b^{(m)}\right)$ abbreviates the array of $B^{(m)}$ parameters

$$
b_{j}^{(m)}\left(j=1, \ldots, B^{(m)}\right) ; \quad \forall m \in\{1, \ldots, n\},
$$

with similar interpretations for $(c)$ and $\left(d^{(m)}\right)(m=1, \ldots, n)$.

The interested reader may refer to papers on the subject for more details $[1,2]$.

The familiar generalized hypergeometric series ${ }_{p} F_{q}$ is defined as (Ref. [3], Section 1.5)

$$
\begin{aligned}
{ }_{p} F_{q}\left[\begin{array}{l}
\gamma_{1}, \ldots, \gamma_{p} ; z \\
\beta_{1}, \ldots, \beta_{q} ;
\end{array}\right] & =\sum_{n=0}^{\infty} \frac{\left(\gamma_{1}\right)_{n} \cdots\left(\gamma_{p}\right)_{n}}{\left(\beta_{1}\right)_{n} \cdots\left(\beta_{q}\right)_{n}} \frac{z^{n}}{n !} \\
& ={ }_{p} F_{q}\left(\gamma_{1}, \ldots, \gamma_{p} ; \beta_{1}, \ldots, \beta_{q} ; z\right),
\end{aligned}
$$

where $(\gamma)_{n}$ is defined as the Pochhammer symbol (for $\gamma \in \mathbb{C}$ ) and it is denoted by [3] (pp. 2, 4-6)

$$
\begin{aligned}
(\gamma)_{n}: & = \begin{cases}1 & (n=0) \\
\gamma(\gamma+1) \ldots(\gamma+n-1) & (n \in \mathbb{N}:=\{1,2,3, \ldots\})\end{cases} \\
& =\frac{\Gamma(\gamma+n)}{\Gamma(\gamma)} \quad\left(\gamma \in \mathbb{C} \backslash \mathbb{Z}_{0}^{-}\right),
\end{aligned}
$$

and $\mathbb{Z}_{0}^{-}$denotes the set of nonpositive integers.

Furthermore, Oberhettinger's integral formula [4]

$$
\int_{0}^{\infty} x^{\mu-1}\left(x+b+\sqrt{x^{2}+2 b x}\right)^{-\eta} d x=2 \lambda b^{-\eta}\left(\frac{b}{2}\right)^{\mu} \frac{\Gamma(2 \mu) \Gamma(\eta-\mu)}{\Gamma(1+\eta+\mu)},
$$

provided $0<\Re(\mu)<\Re(\eta)$.

The well-known Mittag-Leffler function and its generalization were introduced and studied by Mittag-Leffler [5,6], Wiman [7,8], Agarwal [9], Humbert [10], Humbert and Agarwal [11] and other authors [12-15].

Motivated by above works here, with the same technique as Choi and Agarwal [12], we propose the establishment of two generalized integral formulas involving a multivariate generalized Mittag-Leffler function, which are expressed in terms of the generalized Lauricella series due to Srivastava and Daoust [1] given in Equation (1).

In a recent paper, Saxena and Kalla [16] introduced a more generalized Mittag-Leffler function as

$$
E_{\left(\rho_{j}\right), \eta}^{\gamma_{j}}\left(z_{1}, \ldots, z_{m}\right) \equiv E_{\left(\rho_{1}, \ldots, \rho_{m}\right), \eta}^{\left(\gamma_{1}, \ldots, \gamma_{m}\right)}\left(z_{1}, \ldots z_{m}\right)=\sum_{k_{1}, \ldots, k_{m}=0}^{\infty} \frac{\left(\gamma_{1}\right)_{k_{1}} \ldots \ldots\left(\gamma_{m}\right)_{k_{m}} z_{1}^{k_{1}} \ldots . . z_{m}^{k_{m}}}{\Gamma\left(\eta+\rho_{1} k_{1}+\ldots .+\rho_{m} k_{m}\right)\left(k_{1}\right) ! \ldots . .\left(k_{m}\right) !},
$$

where $\eta, \gamma_{j}, \rho_{j}, z_{j} \in \mathbb{C}, \Re\left(\rho_{j}\right)>0, j=1, \ldots, m$.

Equation (7) is a generalization of well-known results.

On setting $m=1$, Equation (7) reduces to the Mittag-Leffler function defined by Prabhakar [15]):

$$
E_{\rho, \eta}^{\gamma}(z)=\sum_{k=0}^{\infty} \frac{(\gamma)_{k} z^{k}}{\Gamma(k \rho+\eta) k !}
$$


where $\rho, \eta, \gamma, z \in \mathbb{C}, \Re(\rho)>0, \Re(\eta)>0$ and $(\eta)_{n}$.

On setting $\gamma=1$, Equation (8) reduces to the Mittag-Leffler function defined by Wiman [8]:

$$
E_{\rho, \eta}(z)=\sum_{k=0}^{\infty} \frac{z^{k}}{\Gamma(k \rho+\eta) k !},
$$

where $\rho, \eta, z \in \mathbb{C}, \Re(\rho)>0, \Re(\eta)>0$,

On setting $\eta=1$, Equation (9) reduces to the Mittag-Leffler function defined by $[5,6]$

$$
E_{\rho}(z)=\sum_{k=0}^{\infty} \frac{z^{k}}{\Gamma(k \rho+1) k !},
$$

where $\rho \in \mathbb{C}, \Re(\rho)>0, z \in \mathbb{C}$.

We also require the generalized hypergeometric function ${ }_{p} \psi_{q}[z]($ see $[17,18])$ defined by

$$
{ }_{p} \psi_{q}[z]=\sum_{k=0}^{\infty} \frac{\prod_{i=1}^{p} \Gamma\left(a_{i}+\alpha_{i} k\right)}{\prod_{j=1}^{q} \Gamma\left(b_{j}+\beta_{j} k\right)} \frac{z^{k}}{k !}
$$

provided that $p, q \in N_{0}=N \bigcup\{0\} ; a_{i}, b_{j} \in \mathbb{C} ; \alpha_{i}, \beta_{j} \in \mathbb{R} ; \alpha_{i}, \beta_{j} \neq 0 ; i=1, \ldots . p ; j=1, \ldots, q$.

\section{Main Results}

We establish two generalized integral formulas, which are expressed in terms of the generalized Lauricella functions (1), by inserting a generalized Mittag-Leffler function (7) with suitable arguments into the integrand of (6).

Theorem 1. The following integral formula holds true: For $\eta, \mu, z_{j} \in \mathbb{C}$ and $x>0$, where $j=1, \cdots, m$

$$
\begin{gathered}
\int_{0}^{\infty} x^{\mu-1}\left(x+b+\sqrt{x^{2}+2 b x}\right)^{-\eta} E_{\left(\rho_{j}\right), \eta}^{\left(\gamma_{j}\right)}\left(\frac{z_{j}}{x+b+\sqrt{x^{2}+2 b x}}\right) d x \\
=2^{1-\mu} b^{\mu-\eta} \frac{\Gamma(1+\eta) \Gamma(\eta-\mu)}{(\Gamma(\eta))^{2} \Gamma(1+\eta+\mu)} \\
\cdot F_{3: 0 ; \ldots ; 0}^{2: 1 ; \ldots ; 1}\left[\begin{array}{l}
{[1+\eta: 1, \ldots, 1],[\eta-\mu: 1, \ldots, 1],} \\
{[\eta: 1, \ldots, 1],[1+\eta+\mu: 1, \ldots, 1],\left[\eta: \rho_{1}, \ldots, \rho_{m}\right]}
\end{array}:\right. \\
\left.\underline{\left[\gamma_{1}: 1\right]} ; \ldots ; \underline{\left[\gamma_{m} ; 1\right]} ; \frac{z_{1}}{b}, \ldots, \frac{z_{m}}{b}\right], \\
-; \ldots ;-
\end{gathered}
$$

where $0<\Re(\mu)<\Re(\eta)$. 
Theorem 2. The following integral formula holds true: For $\eta, \mu, z_{j} \in \mathbb{C}$ and $x>0$, where $j=1, \cdots, m$

$$
\begin{gathered}
\int_{0}^{\infty} x^{\mu-1}\left(x+b+\sqrt{x^{2}+2 b x}\right)^{-\eta} E_{\left(\rho_{j}\right), \eta}^{\left(\gamma_{j}\right)}\left(\frac{x z_{j}}{x+b+\sqrt{x^{2}+2 b x}}\right) d x \\
=2^{1-\mu} a^{\mu-\eta} \frac{\Gamma(\eta-\mu) \Gamma(1+\eta)}{(\Gamma(\eta))^{2} \Gamma(1+\eta+\mu)} \\
\cdot F_{3: 0 ; \ldots ; 0}^{2: 1 ; \ldots ;}\left[\begin{array}{r}
{[1+\eta: 1, \ldots, 1],[2 \mu: 2, \ldots, 2],} \\
{[\eta: 1, \ldots, 1],[1+\eta+\mu: 2, \ldots, 2],\left[\eta: \rho_{1}, \ldots, \rho_{m}\right]}
\end{array}\right. \\
\left.\frac{\left[\gamma_{1}: 1\right]}{;} ; \ldots ; \frac{\left[\gamma_{m} ; 1\right]}{-;} ; z_{1}, \ldots, \frac{z_{m}}{2}\right],
\end{gathered}
$$

where $0<\Re(\mu)<\Re(\eta)$.

Proof. For convenience, let the left-hand side of the assertion (12) be denoted by $\mathcal{I}$. By applying (7) to the integrand of (12), we obtain

$$
\begin{aligned}
\mathcal{I}= & \int_{0}^{\infty} x^{\mu-1}\left(x+b+\sqrt{x^{2}+2 b x}\right)^{-\eta} \\
& \cdot \sum_{k_{1}, \cdots, k_{m}=0}^{\infty} \frac{\left(\gamma_{1}\right)_{k_{1}} \ldots\left(\gamma_{m}\right)_{k_{m}}}{\Gamma\left(\eta+\rho_{1} k_{1}+\ldots+\rho_{m} k_{m}\right)}\left(\frac{z_{1}}{x+b+\sqrt{x^{2}+2 b x}}\right)^{k_{1}} \frac{1}{k_{1} !} \cdots\left(\frac{z_{m}}{x+b+\sqrt{x^{2}+2 b x}}\right)^{k_{m}} \frac{1}{k_{m} !} d x
\end{aligned}
$$

Then, interchanging the order of integration and summation,

$$
\mathcal{I}=\sum_{k_{1}, \cdots, k_{m}=0}^{\infty} \frac{\left(\gamma_{1}\right)_{k_{1}} \ldots\left(\gamma_{m}\right)_{k_{m}}}{\Gamma\left(\eta+\rho_{1} k_{1}+\ldots+\rho_{m} k_{m}\right)} \frac{z_{1}^{k_{1}}}{k_{1} !} \cdots \frac{z_{m}^{k_{m}}}{k_{m} !} \int_{0}^{\infty} x^{\mu-1}\left(x+b+\sqrt{x^{2}+2 b x}\right)^{-\left(\eta+k_{1}+\cdots k_{m}\right)} d x
$$

we can apply the integral formula (6) to the integral in (14) and obtain the following expression:

$$
\begin{aligned}
\mathcal{I}= & \sum_{k_{1}, \ldots, k_{m}=0}^{\infty} \frac{\left(\gamma_{1}\right)_{k_{1}} \ldots\left(\gamma_{m}\right)_{k_{m}}}{\Gamma\left(\eta+\rho_{1} k_{1}+\ldots+\rho_{m} k_{m}\right)} \\
& 2\left(\eta+k_{1}+\cdots+k_{m}\right) b^{-\left(\eta+k_{1}+\ldots+k_{m}\right)}\left(\frac{b}{2}\right)^{(\mu)} \frac{\Gamma(2 \mu) \Gamma\left(\eta+k_{1}+\ldots+k_{m}-\mu\right)}{\Gamma\left(1+\eta+\mu+k_{1}+\cdots+k_{m}\right)} \frac{\left(z_{1}\right)^{k_{1}}}{k_{1} !} \ldots \frac{\left(z_{m}\right)^{k_{m}}}{k_{m} !}
\end{aligned}
$$

Now, arranging the constant term and using $\eta+k_{1}+\cdots+k_{m}=\frac{\Gamma\left(\eta+k_{1}+\cdots+k_{m}+1\right)}{\Gamma\left(\eta+k_{1}+\cdots+k_{m}\right)}$, we obtain

$$
\begin{gathered}
\mathcal{I}=2^{1-\mu} b^{\mu-\eta} \Gamma(2 \mu) \sum_{k_{1}, \ldots, k_{m}=0}^{\infty} \frac{\left(\gamma_{1}\right)_{k_{1}} \ldots\left(\gamma_{m}\right)_{k_{m}}}{\Gamma\left(\eta+\rho_{1} k_{1}+\ldots+\rho_{m} k_{m}\right)} \frac{\Gamma\left(\eta+k_{1}+\cdots+k_{m}+1\right)}{\Gamma\left(\eta+k_{1}+\cdots+k_{m}\right)} \\
\frac{\Gamma\left(\eta+k_{1}+\cdots+k_{m}-\mu\right)}{\Gamma\left(1+\eta+\mu+k_{1}+\cdots+k_{m}\right)}\left(\frac{z_{1}}{b}\right)^{k_{1}} \frac{1}{k_{1} !} \cdots\left(\frac{z_{m}}{b}\right)^{k_{m}} \frac{1}{k_{m} !} .
\end{gathered}
$$

The above equation can be multiplied and devided with $\Gamma(\eta+1),(\Gamma(\eta))^{2}, \Gamma(\eta-\mu)$, $\Gamma(1+\eta+\mu)$ 
Now, using the properties of the Gamma function as $(1+\eta)_{k_{1}+\cdots+k_{m}}=\frac{\Gamma\left(1+\eta+k_{1}+\cdots+k_{m}\right)}{\Gamma(1+\eta)}$, we find that

$$
\begin{aligned}
\mathcal{I}= & 2^{1-\mu} b^{\mu-\eta} \Gamma(2 \mu) \frac{\Gamma(1+\eta)}{\Gamma(\eta)} \frac{1}{\Gamma(\eta)} \frac{\Gamma(\eta-\mu)}{\Gamma(1+\eta+\mu} \\
& \cdot \sum_{k_{1}, \cdots, k_{m}=0}^{\infty} \frac{(1+\eta)_{k_{1}+\cdots+k_{m}}(\eta-\mu)_{k_{1}+\cdots+k_{m}}}{(\eta)_{k_{1}+\cdots+k_{m}}(1+\eta+\mu)_{k_{1}+\cdots+k_{m}}} \\
& \cdot \frac{(\gamma)_{k_{1}} \cdots(\gamma)_{k_{m}}}{(\eta)_{\rho_{1} k_{1}+\cdots+\rho_{m} k_{m}}} \frac{\left(z_{1} / b\right)^{k_{1}}}{k_{1} !} \cdots \frac{\left(z_{m} / b\right)^{k_{m}}}{k_{m} !} .
\end{aligned}
$$

Finally, we interpret the multiple series in (15) as a special case of the general hypergeometric series in several variables defined by (1). Thus, we are led to the assertion (12). The assertion (13) of the Theorem 2.2 can be proved by a similar argument.

\section{Special Cases}

In this section, we derive certain new integral formulas involving Prabhakar-type Mittag-Leffler functions [15] in the integrands of (12) and (13), respectively.

By setting $m=1$ in (12) and (13) and applying the expression in (1) to the identities, we obtain two integral formulas, as stated in Corollary 1 and 2, respectively.

\section{Corollary 1.}

$$
\begin{aligned}
& \int_{0}^{\infty} x^{\mu-1}\left(x+b+\sqrt{x^{2}+2 b x}\right)^{-\eta} \\
& \quad \cdot E_{\rho, \eta}^{\gamma}\left(\frac{z}{x+b+\sqrt{x^{2}+2 b x}}\right) d x=\frac{\Gamma(2 \mu)}{\Gamma(\gamma)} 2^{(1-\mu)} a^{(\mu-\eta)}{ }_{3} \psi_{3}\left[\begin{array}{l}
(\gamma, 1),(\eta-\mu, 1),(\eta+1,1) ; \\
(\eta, \rho),(1+\eta+\mu, 1),(\eta, 1) ;
\end{array}\right]
\end{aligned}
$$

with the convergence conditions followed by Theorem 1.

\section{Corollary 2.}

$$
\begin{aligned}
& \int_{0}^{\infty} x^{\mu-1}\left(x+b+\sqrt{x^{2}+2 b x}\right)^{-\eta} \\
& \quad \cdot E_{\rho, \eta}^{\gamma}\left(\frac{x z}{x+b+\sqrt{x^{2}+2 b x}}\right) d x=\frac{\Gamma(\eta-\mu)}{\Gamma(\gamma)} 2^{(1-\mu)} b^{(\mu-\eta)}{ }_{3} \psi_{3}\left[\begin{array}{c}
(\gamma, 1),(\gamma+1,1),(2 \mu, 2) ; \\
(\eta, \rho),(1+\eta+\mu, 2),(\eta, 1) ;
\end{array}\right],
\end{aligned}
$$

with the convergence conditions followed by Theorem 2 .

It is easily seen that, if we set $\gamma=1$ in (16) and (17), we obtain new integral formulas, as stated in Corollary 3 and 4 , respectively.

\section{Corollary 3.}

$$
\begin{aligned}
\int_{0}^{\infty} x^{\mu-1}\left(x+b+\sqrt{x^{2}+2 b x}\right)^{-\eta} \\
\quad \cdot E_{\rho, \eta}\left(\frac{z}{x+b+\sqrt{x^{2}+2 b x}}\right) d x=2^{(\mu-1)} b^{(\mu-\eta)} \Gamma(2 \mu)_{2} \psi_{3}\left[\begin{array}{r}
(\eta-\mu, 1),(\eta+1,1) ; \\
(\eta, \rho),(1+\eta+\mu, 1),(\eta, 1) ;
\end{array}\right],
\end{aligned}
$$

with the convergence conditions followed by Theorem 1. 


\section{Corollary 4.}

$$
\begin{aligned}
& \int_{0}^{\infty} x^{\mu-1}\left(x+b+\sqrt{x^{2}+2 b x}\right)^{-\eta} \\
& \quad \cdot E_{\rho, \eta}\left(\frac{x z}{x+b+\sqrt{x^{2}+2 b x}}\right) d x=2^{(\mu-1)} b^{(\mu-\eta)} \Gamma(\eta-\mu)_{2} \psi_{3}\left[\begin{array}{r}
(\eta+1,1),(\eta+1,1) ; \\
(\eta, \rho),(1+\eta+\mu, 2),(\eta, 1) ;
\end{array}\right],
\end{aligned}
$$

with the convergence conditions followed by Theorem 2 .

\section{Conclusions}

We conclude our investigation by remarking that the results presented here can be easily converted in terms of the known and new integral formulas after small changes in parameters. We are investigating the main results to find potentially useful applications in a variety of areas.

Author Contributions: Formal analysis, S.J.; funding acquisition, P.A., R.P.A. and S.J.; investigation, S.J., P.S. and P.A.; methodology, S.J., P.S. and P.A.; project administration, R.P.A., S.J. and P.A.; resources, S.J.; supervision, P.A.; Writing—original draft, S.J. and P.S.; Writing—review and editing, R.P.A. and P.A. All authors have read and agreed to the published version of the manuscript.

Funding: S.J. is very thankful to the funding agency SERB (project number: MTR/2017/000194) for providing necessary financial support for the present study.

Institutional Review Board Statement: Not applicable.

Informed Consent Statement: Not applicable.

Data Availability Statement: Not applicable.

Acknowledgments: The authors would like to thank the anonymous referees for their careful reading of this manuscript and also for their constructive suggestions which considerably improved the article. S.J. very thankful to SERB (project number: MTR/2017/000194) for providing necessary facility.

Conflicts of Interest: The authors declare no conflict of interest.

\section{References}

1. Srivastava, H.M.; Daoust, M.C. A note on the convergence of Kampé de Fériet's double hypergeometric series. Math. Nachr. 1985, 53, 151-159. [CrossRef]

2. Srivastava, H.M.; Exton, H. A generalization of the Weber-Schafheitlin integral. J. Reine Angew. Math. 1979, 309, 1-6.

3. Srivastava, H.M.; Choi, J. Zeta and q-Zeta Functions and Associated Series and Integrals; Elsevier: Amsterdam, The Netherlands; London, UK; New York, NY, USA, 2012.

4. Oberhettinger, F. Tables of Mellin Transforms; Springer: New York, NY, USA, 1974.

5. Mittag-Leffler, G.M. Sur la nouvelle fonction $E_{\alpha}(x)$. CR Acad. Sci. Paris 1903, 137, 554-558.

6. Mittag-Leffler, G.M. Sopra la funzione $E_{\alpha}(x)$. Rend. Della R. Accad. Lincei Ser. V 1904, 13, 3-5.

7. Wiman, A. Über den Fundamental satz in der Theorie der Funcktionen, $E_{\alpha}(x)$. Acta Math. 1905, 29, 191-201. [CrossRef]

8. Wiman, A. Über die Nullstellun der Funktionen $E_{\alpha}(x)$. Acta Math. 1905, 29, 217-234. [CrossRef]

9. Agarwal, R.P. A propos d̀une note de M. Pierre Humbert. CR Acad. Sci. Paris 1953, 236, 2031-2032.

10. Humbert, P. Quelques resultants retifs a la fonction de Mittag-Leffler. CR Acad. Sci. Paris 1953, 236, 1467-1468.

11. Humbert, P.; Agarwal, R.P. Sur la fonction de Mittag-Leffler et quelques unes de ses generalizations. Bull. Sci. Math. Ser. II 1953, $77,180-185$.

12. Choi, J.; Agarwal, P. Certain unified integrals involving a product of Bessel functions of the first kind. Honam Math. J. 2013, 35, 667-677. [CrossRef]

13. Milovanović, G.V.; Rassias, M.T. Analytic Number Theory, Approximation Theory, and Special Functions; Springer: New York, NY, USA, 2014.

14. Gorenflo, R.; Kilbas, A.A.; Mainardi, F.; Rogosin, S.V. Mittag-Leffler Functions, Related Topics and Applications, 2nd ed.; Springer: New York, NY, USA, 2020.

15. Prabhakar, T.R. A singular integral equation with a generalized Mittag-Leffler function in the kernel. Yokohama Math. J. 1971, 19, 7-15.

16. Saxena, R.K.; Kalla, S.L. Solutions of Volterra-type integro-differential equations with a generalized Lauricella confluent hypergeometric function in the kernels. Int. J. Math. Math. Sci. 2005, 8, 1155-1170. [CrossRef]

17. Wright, E.M. The asypmtotic expansion of the generalized hypergeometric function. J. Lond. Math. Soc. 1935, 10, $287-293$.

18. Kiryakova, V. Generalized Fractional Calculus and Applications; Longman: Harlow, UK; Wiley: New York, NY, USA, 1994. 